\title{
P04-5-1 Poster session
}

\section{Modification with SUMO to regulate HDAC2 deacetylation activity by carbocisteine in human tracheobronchial epithelial cells}

\author{
Yongyao Cui, Yun Song, Ping Yu, Juan Li, Hong-Zhuan Chen \\ Department of Pharmacology, Shanghai Jiao Tong University School of Medicine, China
}

Background: Post-translational modifications (PTM) of histone deacetylase 2 (HDAC2), including SUMO-mediated sumoylation, determine HDAC2 activity. Our previous studies have shown that cigarette smoke extract (CSE)-induced oxidative stress decreased HDAC2 expression/activity and thus stimulated gene expression of inflammatory factors. Methods. $16 \mathrm{HBE}$ cells were used as the in vitro model to investigate the role of S-CMC as well as the underlying mechanisms. Results:Carbocisteine (S-CMC) blocked this oxidative and subsequently enhanced sensitivity of steroid therapy. The current study was to investigate the molecular mechanisms by which CSE and S-CMC regulate HDAC2 activity and IL-8 expression that mediates inflammatory responses in human tracheobronchial epithelial cells. Modification of HDAC2 by SUMO1 and SUMO2/3 occurred in $16 \mathrm{HBE}$ cells under physiological conditions, and CSE induced SUMO1-mediated modification of HDAC2 in a dose and time-dependent manner. K462 and K51 of HDAC2 were the two major modification sites of SUMO1, and the K51 site mediated deacetylation function of HDAC2 on histone H4 that regulates IL-8 secretion. S-CMC inhibited CSE-induced SUMO1 modification of HDAC2 in the presence of thiol/GSH, leading to deacetylation of histone H4 and decreased IL-8 expression. Conclusions:Our studies may reveal molecular mechanisms that mediate S-CMC-induced sensitivity of steroid treatment of oxidative stress in chronic obstructive pulmonary disease. 